\title{
Hare or Tortoise? Trade-offs in Recovering Sustainable Bioeconomic Systems
}

\author{
Vincent Martinet ${ }^{\mathrm{a},{ }^{*},}$, Olivier Thébaud ${ }^{\mathrm{b}}$, Alain Rapaport ${ }^{\mathrm{c}}$ \\ ${ }^{a}$ Economie Publique, UMR INRA-AgroParisTech., Av. Brétignières, 78850 Thiverval-Grignon, France \\ ${ }^{\mathrm{b}}$ IFREMER, UMR AMURE, Brest, France, and CSIRO Marine and Atmospheric Research, Cleveland, \\ Queensland, Australia \\ ' INRA, UMR INRA-SupAgro MISTEA (Mathématiques, Informatique et STatistique pour l'Environnement et \\ l'Agronomie), Montpellier, France \\ *: Corresponding author : V. Martinet, email address : vincent.martinet@grignon.inra.fr
}

\begin{abstract}
:
In this paper, we develop a framework for (a) the study of sustainability of dynamic bioeconomic systems and (b) the definition of recovery paths from unsustainable situations. We assume that the system follows a sustainable trajectory if it evolves over time within a set of multidimensional constraints. We use the mathematical concept of viability to characterize sustainability. Recovery paths are studied with regards to their duration and their acceptability. This general framework is applied to the issue of recovering sustainable fisheries. We define sustainability in a fishery as the requirement that a set of economic, ecological, and social constraints is satisfied at all times. Recovery paths are characterized by the time required to obtain sustainable exploitation conditions in the fishery and by the acceptable recovery costs for fishermen. In particular, we identify the recovery path which minimizes the time of crisis under a minimum transition profit constraint. We then describe the tradeoff between speed and accepted costs of recovery paths, by comparing "Hare"-like high-speed-highcost strategies to "Tortoise"-like low-speed-low-cost strategies. We illustrate our results by means of a numerical analysis of the Bay of Biscay Nephrops fishery.
\end{abstract}

Keywords: Sustainability - Recovery strategies - Multicriteria approach - Optimal and viable control Bioeconomic modeling - Fisheries economics 


\section{Introduction}

This paper proposes a framework to define sustainability and to analyze recovery processes leading to sustainable bioeconomic systems. As this work is motivated by the issue of over-exploitation of natural resources, we will refer to this concern to illustrate our general results. Nowadays the sustainable management of natural renewable resources is a primary policy objective, at both national and international levels ([39]). However, as many resources are already overexploited ([30]), the need to define recovery paths toward sustainable exploitation patterns has become more critical. To address this, one must understand the interactions between ecological and economic dynamics. Bioeconomic modeling offers a powerful way to describe and explore the implications of these interactions. We develop a general framework to study the restoration of bioeconomic systems to sustainable exploitation configurations, and illustrate our results by means of an application to the specific issue of recovering sustainable fisheries, starting from unsustainable situations.

Following the analysis by Charles [4], we adopt a multi-criteria approach to the definition of sustainability in fisheries. According to Cochrane [10], the primary considerations in fisheries management are sustainability of the resource base, economic viability and equity in access to the resource. When one of the ecological, economic or social objectives is not met, fisheries face a crisis/unsustainable situation. One of the reasons for management failure in fisheries is the conflict between ecological constraints and social and economic priorities; the latter often take priority over resource conservation $([20])$, leading to crisis situations. A challenge for fisheries management models is thus to develop approaches that meet diverse sustainability objectives. Such approaches have been advocated by Mardle and Pascoe [26] based on the use of multicriteria decision-making methods. In economic theory, the usual approach is to use multi-objective optimization, which requires the identification and formalization of a function embodying all the criteria to need to be maximized. This can be achieved, for instance, by defining a set of weights for those criteria in order to materialize the trade-offs between conflicting objectives (e.g., resource biomass, yield, revenues, employment). For example, [5] describe objectives in fisheries management, and trade-offs in their achievement in a simulation framework, using arbitrary weighting factors for policy objectives. However, determining such weights may be difficult as it involves trade-offs implying normative choices regarding the relative importance of different objectives, and it often depends on the relative bargaining power of stakeholders. Alternatively, conflicting management objectives could be accounted for by using indicators, with objectives defined by thresholds. While such thresholds may be difficult to identify and agree upon, they may prove to be easier to establish than the actual weights on management objectives. If sustainability objectives are so defined, the regulator's problem becomes to 
avoid situations in which some indicators do not reach the thresholds representing sustainability objectives. Sustainability would then appear to be more a 'satisficing' problem $([25,35])$ than an optimization one. The role of scientists in such an approach may be to identify the different decisions which satisfy all the sustainability requirements. From this perspective, the status of a fishery can be characterized by a set of indicators, with thresholds defined as constraints reflecting the different objectives of fishery management. Sustainability is then defined as the ability of the fishery to evolve, in the long-run, within the specified set of constraints.

Viability theory ([1]) is particularly well-suited to addressing such problems. This theory relies on the definition of a set of constraints that must be satisfied at all times for a system to be said to be viable, and on the study of the consistency between the dynamics of the system and these constraints. This results in the definition of the so-called viability kernel of the problem, which is the set of initial bioeconomic states for which there are decisions that result in intertemporal trajectories satisfying all the constraints. This framework has been used in [25] to study the viability of monetary policies in a macroeconomic context, and in [28] to study the sustainability of an economy with an exhaustible resource. The viability of bioeconomic systems with renewable resources has been studied by several authors since the publication by [2] (see [12] and references therein). Cury et al. [11] advocated viability analysis in the context of the Ecosystem Approach to Fisheries management.

According to recent studies, there has been an increase in the proportion of over- exploited marine fish stocks world-wide, and excess-fishing capacities exist in most fisheries $([15,16])$. Hence, the problem of managing fisheries is increasingly set in terms of restoring fish stocks, catches, and revenues to sustainable levels. From an economic point of view, the optimal recovery strategy is often related to a "most rapid approach" which may require the closure of the fishery ([7]). This may not be acceptable for fishermen, and difficult to apply in practice due to the short-term economic and social implications of such transition paths. While the viability framework defines sustainable exploitation configurations, it does not deal with recovery problems. Doyen and Saint-Pierre [13] extend the viability approach to deal with such issues, and define the notion of minimum time of crisis. Martinet et al. [29] use this notion to analyze recovery paths for bioeconomic marine resource systems facing crisis situations. They compare a recovery strategy based on the minimization of the time of crisis to other possible strategies (historical path, Open access and Maximum Intertemporal Economic Yield). The minimum time of crisis strategy also requires closure of the fishery during the recovery process, which entails high short term costs to fishing operators. If there are no alternative sources of revenue or employment available for fishermen, such strategies are bound to be met with stiff resistance, as stressed by Cochrane [10]. In practice, this may seriously compromise the feasibility of recovery strategies. There- 
fore, the successful implementation of a recovery program may be conditioned by the reduction of economic losses suffered by individual operators during transition phases. Hence, these programs have two important dimensions: i) achieving sustainability as quickly as possible, while ii) defining recovery paths with acceptable costs to individual operators. There will necessarily be tradeoffs between the speed of recovery and the social and political acceptability of the adjustments required for fisheries to be restored. This problem is not addressed in Martinet et al. [29].

In the present article, we extend the analysis of Martinet et al. [29] to address the issue of the restoration of bioeconomic systems facing a crisis. More specifically, we examine the trade-offs between speed and acceptability of recovery programs. The analysis is based on a bioeconomic model representing the dynamics of a fishery, and the definition of a set of constraints describing sustainability boundaries for this fishery. The objective of the social planner is to keep the fishery within these boundaries in the long-run. The fishery will be said to be confronted with a crisis if this objective is not met. Three categories of constraints are considered: biological, economic and social, without prioritizing any one dimension over the others $([18,32])$. We first use viability theory to identify the set of initial states of the fishery from which it is possible to satisfy these constraints dynamically. We then consider the problem faced by a social planner who seeks to return the fishery to a satisfactory state when it is not initially in that set of states. We treat this as a specific optimal control problem in which the social objective is to minimize the duration of crisis under constraints related to the acceptability of transition costs. These transition costs are defined as the costs incurred by individual operators due to the implementation of a recovery program. We consider that these costs cannot be higher than a given acceptable level. Treating this level as a parameter, we define the minimum time of crisis with respect to this parameter (resulting in the definition of a parameterized value function), which allows us to quantify trade-offs between the duration of crisis and the acceptability of recovery programs. In particular, we describe two extreme and opposing strategies which we define as the Hare or the Tortoise strategy. Choosing to act like a Hare would consist in planning the quickest possible recovery, whatever the individual cost. This may result in unacceptable recovery programs that would be postponed. Choosing to act as a tortoise would consist in reducing recovery speed in order to satisfy short term requirements, to limit individual costs, and should result in a well-accepted recovery program, but with the risk that recovery may not be possible in finite time.

To illustrate this general problem, we base our analysis on a previous empirical application ([29]) which considered a discrete time bio-economic model, with two state variables (resource stock biomass and fleet size) and two control variables (per vessel fishing effort and fleet adjustment speed) to represent the Bay of Biscay (ICES area VIII) Nephrops fishery. We focus on recovery paths 
from a historical crisis situation. Based on our general framework, we compare various recovery paths to the observed evolution of the fishery.

The article is organized as follows. The general framework, which allows us to study recovery processes for bioeconomic models, is presented in section 2 . The mathematical details for the computation of the problem are presented in Appendix A. In section 3, we present the case study, and describe the bioeconomic model representing the fishery and the viability constraints defining its sustainability. Parameter values and estimation methods are detailed in Appendix B. We describe how the theoretical framework presented in section 2 is applied to characterize viable and crisis situations in our case study. The optimization program is then presented and used to define recovery strategies that minimize the time of crisis under a transition profit constraint. This framework is applied to simulate recovery paths from the historical crisis situation considered in the case study. We conclude in Section 4.

\section{A theoretical framework to define recovery paths toward sus- tainable resource use}

In this section, we develop a general theoretical framework to address the issue of recovery toward the sustainable use of natural resources.

We consider a discrete time dynamic system where the economy is represented at time $t \in \mathbb{N}$ by a vector of $n$ capital stocks $X_{t} \in \mathbb{R}^{n}$. Each capital stock can alternatively be man-made reproducible capital or a natural resource stock. The dynamics of capital stocks is controlled by a vector of $m$ decisions $u_{t} \in$ $\mathcal{U}\left(X_{t}\right) \subset \mathbb{R}^{m}$, where $\mathcal{U}\left(X_{t}\right)$ is the set of feasible controls available to the decision maker, depending on the current state of the system. The capital stocks thus evolve according to the dynamics $F: \mathbb{R}^{n} \times \mathbb{R}^{m} \mapsto \mathbb{R}^{n}$

$$
X_{t+1}=F\left(X_{t}, u_{t}\right) .
$$

We consider that sustainability in this economy encompasses different issues (economic, social or environmental) represented by a set of constraints $\mathcal{S} \subset \mathbb{R}^{n} \times \mathbb{R}^{m}$. This set of constraints represents the desired configurations of the economy. From an intergenerational perspective, we are interested in trajectories that satisfy the constraints at all times.

To analyze the sustainability of the economy, we refer to the viability approach ([1]). We examine the consistency between the dynamics defined by eq. (1) and the constraints set $\mathcal{S}$. Firstly, we define the economic states $X_{0}$ from which there is at least one viable path $X()=.\left(X_{t_{0}}, X_{t_{0}+1}, \ldots\right)$ defined by a sequence of decisions $u()=.\left(u_{t_{0}}, u_{t_{0}+1}, \ldots\right)$ such that the constraints are respected 
dynamically, i.e., $\left(X_{t}, u_{t}\right) \in \mathcal{S}$ for all $t \in \mathbb{N}$. The set of all viable initial states is called the viability kernel of the problem, and denoted

$$
\operatorname{Viab}_{F}(\mathcal{S})=\left\{\begin{array}{l|l}
X_{0} \in \mathbb{R}^{n} & \begin{array}{l}
\exists(X(.), u(.)) \text { starting from } X_{0} \\
\text { and satisfying eq. (1) } \\
\text { such that }\left(X_{t}, u_{t}\right) \in \mathcal{S}, \forall t \in \mathbb{N}
\end{array}
\end{array}\right\} .
$$

Secondly, we identify the sequences of controls that allow the economy to remain within the constraint set $\mathcal{S}$. By definition, from any state within the viability kernel, there is at least one viable decision which makes it possible to meet the viability constraints in the long run; if we denote $\mathcal{U}_{\text {viab }}(X)$ the set of viable decisions associated with a particular viable state $X$, then $X \in$ $\operatorname{Viab}_{F}(\mathcal{S}) \Longrightarrow \mathcal{U}_{\text {viab }}(X) \neq \emptyset$. Note that from states belonging to the viability kernel, there may be admissible decisions $u_{t} \in \mathcal{U}(X)$ that are not viable, which means that the set of viable decisions may be a strict subset of the admissible decisions.

If the states and decisions do not satisfy the constraints, i.e., $\left(X_{t}, u_{t}\right) \notin \mathcal{S}$, the economy is said to be facing a crisis. A direct consequence of the definition of the viability kernel is that, from any state outside the kernel, there is no sequence of decisions that will satisfy the constraints in the long run: at least one of the constraints will be violated in finite time, whatever decisions are made. The system will eventually face a crisis situation if the state is located outside the kernel. Hence, as proved by [1], a trajectory $(X(),. u()$.$) will respect$ the constraints $\mathcal{S}$ only if the state of the system remains within the viability kernel. Sustainability of the economy is thus characterized by the set $\operatorname{Viab}_{F}(\mathcal{S})$ and associated viable decisions $\mathcal{U}_{v i a b}(X)_{\mid X \in \operatorname{Viab}_{F}(\mathcal{S})}$. Given dynamics (1) and admissible sets $\mathcal{U}(X)$, any path starting from states of the economy outside the viability kernel will eventually lead to a crisis.

The duration of a crisis can be formally defined by following [13], who introduce the concept of time of crisis and study its mathematical links with the viability analysis. This concept is used in [2] to analyze bioeconomic crisis situations.

We introduce the crisis indicator

$$
\mathbf{1}_{\mathcal{S}}(X, u)= \begin{cases}0 & \text { if }(X, u) \in \mathcal{S} \\ 1 & \text { otherwise }\end{cases}
$$

This indicator represents the effectiveness of a given configuration of the economy (state and decision) to respect the constraints. For a given trajectory $(X(),. u()$.$) starting from X_{0}$, we define the time of crisis as the number of periods during which the constraints are not met. We write 


$$
\begin{aligned}
& \mathcal{T}(X(.), u(.))=\sum_{t=0}^{\infty} \mathbf{1}_{\mathcal{S}}\left(X_{t}, u_{t}\right) . \\
\text { s.t. } & X_{t+1}=F\left(X_{t}, u_{t}\right) \\
& X_{t=0}=X_{0}
\end{aligned}
$$

Note that a viable trajectory will have a null time of crisis, i.e., will satisfy the constraints forever, and that a trajectory starting outside the viability kernel will have a positive time of crisis.

In this paper, we assume that the objective of the social planner is to minimize the occurrence of crisis situations. When it is possible, the objective will be to satisfy the sustainability constraints at all times, by following a viable trajectory. However, we shall also consider the case in which the social planner is faced with a crisis situation and wants to recover a sustainable economy. Based on the above definition of sustainability, recovering from a crisis situation demands that the economy reaches the viability kernel in the future. As the economy is initially in a non-viable state, this will only be possible if at least one of the constraints is not satisfied during some period of time along the transition path. We assume that the social objective is to minimize the time during which this will occur. ${ }^{1}$

We use the time of crisis defined by eq. (4) as a criterion to be minimized in order to leave a crisis situation and achieve a sustainable economy. With no further considerations, the optimal trajectory would minimize the time of crisis without accounting for violation of the constraints along the recovery path, as in [29]. However, as stressed in the introduction, the social planner may also be concerned by the social acceptability of the recovery program. This may lead to the imposition of restrictive conditions on feasible recovery paths. To take this issue into account, one can define a reduced set of admissible transition decisions $\mathcal{U}_{\text {trans }}(X) \subseteq \mathcal{U}(X)$ representing the set of socially acceptable controls. ${ }^{2}$ For example, the set of admissible decisions during recovery process $\mathcal{U}_{\text {trans }}(X)$ can be defined in relation to annual acceptable costs for individual operators in the economy.

To formalize this approach we define a specific optimal control problem, which minimizes the time of crisis under transition constraints:

\footnotetext{
1 This approach is akin to the "basic needs" analysis of Chichilnisky [6]. The model has the same methodological foundations as the theory of optimal economic growth in that the efficient economic paths are solutions of a constrained optimization problem.

2 Taking $\mathcal{U}_{\text {trans }}(X)=\mathcal{U}(X)$ will lead to the minimum time of crisis without transition constraints. This special case is examined in the context of the fishery in [29].
} 


$$
\begin{aligned}
\mathcal{C}_{\mathcal{U}_{\text {trans }}}\left(X_{0}\right)= & \inf _{u(.)} \mathcal{T}(X(.), u(.)) \\
\text { s.t. } & X_{t+1}=F\left(X_{t}, u_{t}\right), \\
& X_{t=0}=X_{0} \\
& u_{t} \in \mathcal{U}_{\text {trans }}\left(X_{t}\right) .
\end{aligned}
$$

The solution to such an optimization problem defines, for any given initial state $X_{0}$, the minimum time of crisis under transition constraints $\mathcal{U}_{\text {trans }}$, and the associated recovery path.

As already noted, the viability kernel of a problem can be defined as the set of states with a nil minimum time of crisis. More broadly, one can define the set of states with a minimum time of crisis equal to $i$. By generalizing, we can define the $I+1$ sets of states with a minimum time of crisis equal to $i=0, \ldots, I$, and draw a map of times of crisis in the state set $\mathbb{R}^{n}$. For a given set of transition constraints $\mathcal{U}_{\text {trans }}$, the value function $\mathcal{C}_{\mathcal{U}_{\text {trans }}}: \mathbb{R}^{n} \mapsto \mathbb{R}$ defines the value of the minimum time of crisis under constraints for all states. ${ }^{3}$

As the time of crisis depends on the transition constraints set $\mathcal{U}_{\text {trans }}$, by computing the times of crisis map for several transition constraints $\mathcal{U}_{\text {trans }}$, our general framework allows us to discuss the trade-offs between the time of crisis and conditions imposed on the transition path (for example, its cost to economic agents) for any state $X \in \mathbb{R}^{n}$. This allows us to compare transition paths, from a given crisis situation $X_{0}$ to sustainability, with respect to their speed and their social acceptability.

\section{Recovering a sustainable fishery}

To illustrate this general approach, we have designed an application, built on a model of the fishery as presented in [29].

3 To determine the minimum time of crisis map, we compute $\mathcal{C}_{\theta}(\cdot)$, an approximation of the value function $\mathcal{C}_{\mathcal{U}_{\text {trans }}}(\cdot)$ for the whole map $\mathbb{R}^{n}$ using the contraction mapping theorem on an operator with a contraction parameter $\theta$ that guarantees the convergence toward $\mathcal{C}_{\theta}(\cdot)$ (see [3]). The algorithm gives a sequence of approximated optimal recovery decisions $u_{\theta}^{\star}(.) \in \mathcal{U}_{\text {trans }}$. There is a trade-off in the choice of parameter $\theta$ between the precision of the algorithm (i.e., $\mathcal{C}_{\theta}(\cdot)$ is closer to $\mathcal{C}_{\mathcal{U}_{\text {trans }}}(\cdot)$ when $\theta$ is closer to one) and the speed of convergence (i.e., the number of iterations for obtaining $\mathcal{C}_{\theta}$ given the computer precision is lower when $\theta$ gets closer to 0 ). The mathematical details and the numerical methods to compute the map of minimum time of crisis are given in appendix A. 


\subsection{The model}

We consider a single stock fishery, characterized for each year $t$ by two state variables: the biomass $B_{t}$ of the exploited resource stock, and the size of the fleet $K_{t}$. The dynamics of the bio-economic system is driven by two control variables: fishing effort $e_{t}$ (days at sea per period and per vessel), and changes in fleet size $\xi_{t}$ (number of boats entering or leaving the fleet).

We use a logistic function to represent the natural growth of the resource stock $([33])$ :

$$
R\left(B_{t}\right)=r B_{t}\left(1-\frac{B_{t}}{B_{\text {sup }}}\right)
$$

where $B_{\text {sup }}$ is the carrying capacity of the ecosystem for the resource stock, and $r$ its intrinsic growth rate.

The fleet is assumed to be homogeneous. Each vessel has the same access to the resource and the same technical features. Total catches are defined by

$$
C_{t}=q B_{t} e_{t} K_{t}
$$

where $q$ represents the catchability of the resource. Combining eqs. (6) and (7), the dynamics of the resource is

$$
B_{t+1}=B_{t}+R\left(B_{t}\right)-C_{t}=B_{t}+r B_{t}\left(1-\frac{B_{t}}{B_{\text {sup }}}\right)-q B_{t} e_{t} K_{t} .
$$

The economic status of the fleet is characterized by per vessel profit, depending on landings $L_{t}$ of the resource defined with respect to the per vessel catches $c_{t}=C_{t} / K_{t}=q B_{t} e_{t}$ and a discard rate $\tau_{d}$ :

$$
L_{t}=\left(1-\tau_{d}\right) q B_{t} e_{t}
$$

Based on these landings, gross return associated to the targeted species is defined as a part $\lambda$ of the vessel's total gross return. ${ }^{4}$ Vessel profit, defined as the difference between gross return and harvesting costs, thus reads

$$
\pi\left(B_{t}, e_{t}\right)=\left(p\left(1-\tau_{d}\right) q B_{t} e_{t}\right) \frac{1}{\lambda}-\left(\omega_{f}+\omega_{v} e_{t}\right)
$$

where $p$ is an exogenous resource price that is considered constant. The parameter $\omega_{f}$ represents fixed costs and $\omega_{v}$ is a per effort unit cost related to the

\footnotetext{
$\overline{4}$ We consider that the gross return generated by other species in the fishery is proportional to the gross return generated by the targeted species. Taking $\lambda=1$ means that the species studied is the only one landed by the fleet.
} 
catch and landing of the species modeled. Thus defined, profit represents the remuneration of production factors (capital and labor) at vessel level.

The production structure is assumed to be flexible, in terms of both capital and labor. The number of vessels $\xi_{t}$ entering or leaving the fleet is a control variable. The size of the fleet evolves according to

$$
K_{t+1}=K_{t}+\xi_{t}
$$

A degree of capital inertia is assumed to exist in the fishery. ${ }^{5}$ Due to technical and regulatory constraints, a maximum number $\xi_{\text {sup }}$ of vessels can enter the fishery in any given time period. In addition, the number of vessels exiting the fleet in any time period cannot exceed $\xi_{\text {inf }}$, due to economic, social and political constraints. Thus,

$$
-\xi_{\text {inf }} \leq \xi_{t} \leq \xi_{\text {sup }}
$$

The level of fishing activity of the fleet (effort per period $e_{t}$ ) on the other hand, can adjust instantaneously, and is bounded only by the technical constraint that effort per vessel cannot exceed a maximum number of days at sea per period $e_{\text {sup }}$ :

$$
0 \leq e_{t} \leq e_{\text {sup }}
$$

\section{Sustainability defined as a set of viability constraints}

Hilborn [21] explores the nature of fishery management objectives. According to him, these belong to four major categories, which are biological, economic, social and political. The first (and oldest) is the biological objective to preserve stocks and ecosystems and maximize their productivity (Maximum Sustainable Yield). The second is the economic objective of maximizing rents (Maximum Economic Yield). The third encompasses social issues such as employment and income distribution, and maintenance of traditional communities, which Hilborn defines as Maximum Job Yield. The fourth concerns the political dimension (that is usually implicit) of fishery management which seeks to avoid conflict.

Based on this typology of management objectives, we propose to define sustainability of the fishery based on a set of biological, economic and social viability constraints, following [29]. We then introduce a political constraint, which relates to the social acceptability of policies designed to maintain or restore a sustainable fishery.

$\overline{5}$ For the analysis of the consequences of capital adjustment limits in fisheries management, see [36]. 
The economic constraint is defined with respect to vessel profitability which must cover the opportunity costs of the production factors (capital, labor, fishing rights) for the fishing unit to be economically viable ([19]). We assume that profit per vessel is required to be greater than the threshold $\pi_{\min }$ :

$$
\pi_{t} \geq \pi_{\min }
$$

In order to preserve the renewable resource and avoid depletion of the stock, the objective of maintaining the resource stock above a minimum threshold $B_{\min }$ is considered:

$$
B_{t} \geq B_{\min }
$$

However, as proved in [29], with realistic parameters values, the profit constraint (14) generates stronger limitations on stock size than the biological constraint (15). Martinet et al. [29] show that a minimum resource stock for fishing activity to be able to satisfy the per vessel profit constraint (14) is given by

$$
B_{t} \geq \underline{B}\left(\pi_{m i n}\right)=\frac{\pi_{m i n}+\left(\omega_{f}+\omega_{v} e_{s u p}\right)}{\frac{p}{\lambda}\left(1-\tau_{d}\right) q e_{\text {sup }}} .
$$

The social constraint is considered here as the preservation of employment and maintenance of coastal fishing communities. For this purpose, we require the number of active vessels to be greater than the threshold $K_{\min }$ ensuring minimum employment and activity in the fishery:

$$
K_{t} \geq K_{\min }
$$

We denote $\mathcal{S}$ to be the set of constraints defined by eqs. (14), (15) and (17):

$$
\mathcal{S}=\left\{\begin{array}{l|l}
(B, K, e, \xi) & \begin{array}{l}
\left(p\left(1-\tau_{d}\right) q B e\right) \frac{1}{\lambda}-\left(\omega_{f}+\omega_{v} e\right) \geq \pi_{m i n}, \\
B \geq B_{\text {min }}, \\
K \geq K_{\text {min }}
\end{array}
\end{array}\right\}
$$

\section{Case study}

To illustrate our approach, the analysis is applied to a model of the Bay of Biscay Nephrops fishery (ICES area VIIIa,b). The data and parameters are described in appendix B. In 2003, the fleet was composed of 235 vessels with an average annual profit of 165,000 Euro. The resource stock was estimated at about 18,600 tons. The average number of days at sea per vessel was 203 . The total catches were estimated at 5,769 tons. 
Based on available data, the minimum profit per vessel $\pi_{\min }$ (i.e., profit ensuring remuneration of production factors at their opportunity costs) was estimated to be 130,000 Euro per year. ${ }^{6}$ A minimum stock size $B_{\text {min }}$ was fixed at 5,000 tons based on the available biological reference point defined in the literature for that resource stock. The minimum fleet size $K_{\min }$ was arbitrarily fixed at 100 vessels, and the maximum speed of entry/exit of vessels in the fleet (respectively $\xi_{\text {sup }}$ and $\xi_{\text {inf }}$ ) at 5 vessels per year.

Viability constraints were thus met in year 2003. However, the bioeconomic model of the fishery shows that in the early 90s, the resource stock decreased, and the associated per-vessel profit dropped below the economic viability constraint. The modelled resource stock reached its lowest level in 1994, at about 14,000 tons, with an associated per-vessel profit of 78,000 Euro. The fishery thus faced a period of crisis (at least from an economic point of view), from which the model shows that it recovered in later years. For illustrative purposes, we apply the analytical framework developed in section 2 to define recovery processes for that fishery, from the bioeconomic state of 1994 .

\subsection{Viability and crisis of the fishery}

\section{Viable bioeconomic states and associated harvesting strategies}

We first define the bioeconomic states of the fishery (combinations of resource stock and fleet size) which are compatible with the viability constraints. The question is to determine whether the dynamics are compatible with the set of constraints. For this purpose, we use the viable control approach and study the consistency between dynamics (8) and (11) and the viability constraints (14), (15) and (17). For our problem, the viability kernel is formally defined by

$$
\text { Viab }=\left\{\begin{array}{l|l}
\left(B_{0}, K_{0}\right) & \begin{array}{l}
\exists(e(.), \xi(.)) \text { and }(B(.), K(.)), \text { starting from }\left(B_{0}, K_{0}\right) \\
\text { satisfying dynamics }(8) \text { and }(11), \\
\text { control constraints (12) and (13), } \\
\text { and constraints (14), (15) and (17) for any } t \in \mathbb{N}^{+}
\end{array}
\end{array}\right\}
$$

\section{Crisis situations}

As stated in section 2, , sustainability goals cannot be achieved from any state

\footnotetext{
$\overline{6}$ This is established based on the 2003 data collected via the annual economic surveys carried out by Ifremer.
} 
outside the viability kernel. We introduce the crisis indicator

$$
\mathbf{1}_{\mathcal{S}}(B, K, e, \xi)= \begin{cases}0 & \text { if }(B, K, e, \xi) \in \mathcal{S} \\ 1 & \text { otherwise. }\end{cases}
$$

To recover from such crisis situations, the bioeconomic state trajectory must reach the viability kernel. This will only be possible if the decisions taken lead to breaking the constraints during a certain period of time, thus allowing the system to recover. Following the general framework proposed in section 2, a recovery path can then be characterized by its time of crisis, i.e., the number of time periods during which the system is violating some of the viability constraints.

Definition 1 We define the time of crisis of a path $(B(),. K()$.$) associated$ with the decisions $(e(),. \xi()$.$) as the sum$

$$
\mathcal{T}(B(.), K(.), e(.), \xi(.))=\sum_{t=0}^{\infty} \mathbf{1}_{\mathcal{S}}\left(B_{t}, K_{t}, e_{t}, \xi_{t}\right) .
$$

\subsection{Analysis of trade-offs when selecting recovery programs}

As stressed by Hilborn ([21]) an implicit but important political objective in fisheries management is to prevent conflicts. When the fishery is in crisis, conflict will develop between stakeholders. Thus, the main political aim of the social planner may be to avoid crisis situations or, when a crisis is unavoidable, to reduce its duration via appropriate recovery programs. However, as already stressed, recovery programs require the violation of at least one of the viability constraints, leading to additional conflicts. In an overexploited fishery for example, a recovery program based on a fishing ban will face strong resistance from fishermen due to the high individual costs of such a policy. As a result, the program may not be politically acceptable. Thus, a realistic definition of recovery programs for fisheries should include a formal representation of the acceptability of violating one or several of the viability constraints during transition phases.

Using the theoretical framework developed in section 2, we formalize this through the following social planner optimization problem.

The minimum time of crisis problem under acceptability constraints Following Martinet et al. [29], we use the concept of minimum time of crisis to study recovery paths from crisis situations. Starting from any given bioeconomic state, the problem consists in defining the decisions which minimize 
the time of crisis for the resulting fishery recovery trajectory. Each state can thus be characterized by this minimum time of crisis. In particular, note that the viability kernel corresponds to the set of states with a nil minimum time of crisis. This means that minimizing the time of crisis will also define the viable bioeconomic states of the fishery. Moreover, by using this concept of minimum time of crisis, we are able to define the notion of viability at scale $T$, characterizing the states of the fishery from which it is possible to obtain a viable exploitation pattern after a transition with $T$ cumulative years of crisis. ${ }^{7}$

Extending the analysis in [29], we introduce a transition profit constraint

$$
\pi_{t} \geq \pi_{\text {trans }}
$$

Such a constraint underlines the fact that, even if the optimal recovery strategy requires closing the fishery for a while ([7],[29]), this is not always possible because it neglects fishermen's needs to cover some fixed costs and/or to ensure a minimum activity and revenue. One may thus require a minimum level of fishing during the transition phase, or more specifically, a minimum remuneration of labor and capital, even if it is lower than the minimum viable profit, i.e., $\pi_{\text {trans }}<\pi_{\min }$.

Hence, we define the set of all admissible recovery decisions for our fishery

$$
\mathcal{U}_{\text {trans }}\left(B, K, \pi_{\text {trans }}\right)=\left\{\begin{array}{l|l}
(e, \xi) \mid \begin{array}{l}
e \in[0, \bar{e}] \text { and } \pi(B, e) \geq \pi_{\text {trans }} \\
\xi_{\text {sup }} \geq \xi \geq \max \left(K_{\text {min }}-K,-\xi_{\text {min }}\right)
\end{array}
\end{array}\right\}
$$

Therefore, the trajectory starting from $\left(B_{0}, K_{0}\right)$ and having the minimum time of crisis under transition conditions $\mathcal{U}_{\text {trans }}$ is defined by the following optimization problem:

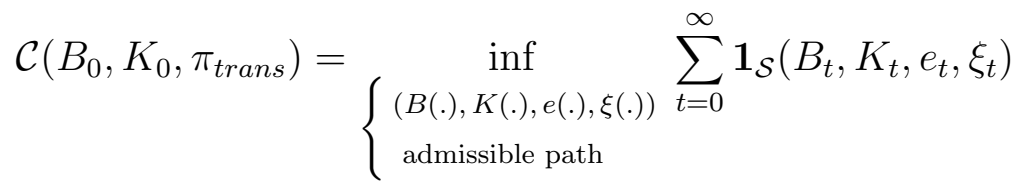

where

- the characteristic function $\mathbf{1}$ is defined by the crisis indicator (19).

- a path $(B(),. K(),. e(),. \xi()$.$) is said to be admissible whenever it satisfies$ dynamics and control constraints (8), (11), (12) and (13) while starting from $\left(B_{0}, K_{0}\right)$, along with the transition profit constraint $(21)$.

\footnotetext{
$\overline{7}$ This corresponds to the set of states for which the minimum time of crisis is lower
} than or equal to $T$. 
Using $\pi_{\text {trans }}$ as a parameter, we can solve this problem for any given transition profit constraint level $\pi_{\text {trans }}$, and represent the trade-off between the minimum time of crisis and the level of $\pi_{\text {trans }}$. Fig 1 represents the viability kernel and the minimum time of crisis for the fishery, under three levels of transition profit constraint. 


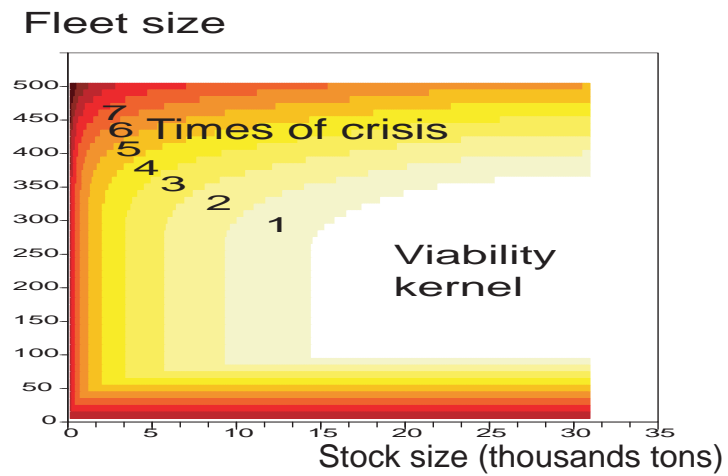

(a) minimum time of crisis without transition profit constraint

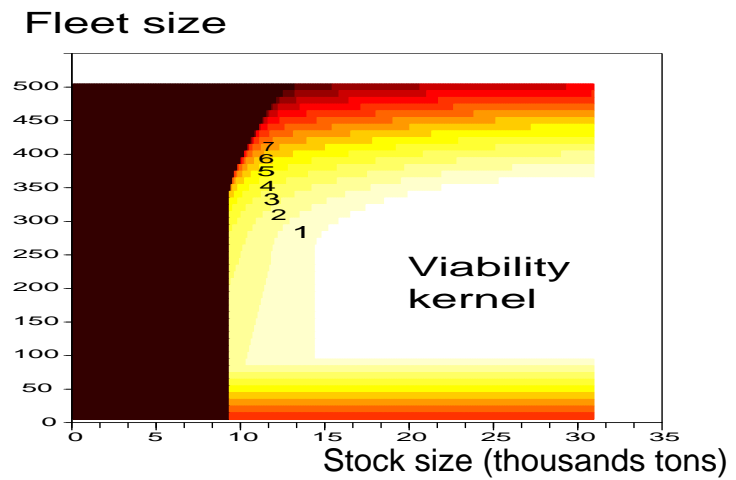

(b) minimum time of crisis with $\pi_{\text {trans }}=30,000$ Euro

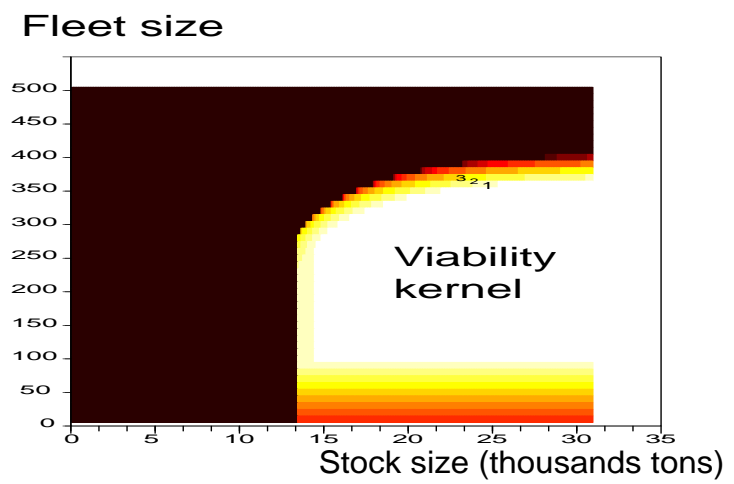

(c) minimum time of crisis with $\pi_{\text {trans }}=$ 110,000 Euro

Fig. 1. Space of bioeconomic states characterized by their minimum time of crisis under various transition constraints. The viability kernel (minimum time of crisis equals to 0) is shown in white. The states in the black area are characterized by an infinite minimum time of crisis. The gradually hotter (darker) colored areas correspond to increasing minimum times of crisis. 
The upper panel (a) of Fig. 1 represents the solution of problem (23) with no constraint on transition profit, as studied in [29]. The central white area represents the viability kernel. The surrounding colored areas correspond to the sets of states with positive minimum times of crisis. Each successive area of hotter (darker) color has a minimum time of crisis of one additional time period (year). The further from the viability kernel the initial state is, the longer it takes to recover from a crisis situation. The central and lower panels of Fig. 1 represent minimum times of crisis corresponding to a recovery program under transition profit constraints: the central panel (b) is produced with $\pi_{\text {trans }}=30,000$ Euro; the lower panel (c) with $\pi_{\text {trans }}=110,000$ Euro. The black area on the left-hand-side of the figures represents states from which it is not possible to recover from the crisis situation given the transition profit constraint and the inherent dynamics of the fishery: bioeconomic states in these areas are associated with an infinite minimum time of crisis. The higher the transition profit constraint, the larger this area. In other words, for any given initial state of crisis of the fishery, there will be a maximum constraint that can be imposed on transition profit for a recovery program to succeed in finite time. Also note that, for any given initial state of the fishery, an increase in this transition constraint will increase the minimum time of crisis: Any given state will belong to an area of the map characterized by a higher (or equal) minimum time of crisis when the guaranteed transition profit increases. Mathematically, this reads $\pi_{1}>\pi_{2} \Rightarrow \mathcal{C}\left(B_{0}, K_{0}, \pi_{1}\right) \geq \mathcal{C}\left(B_{0}, K_{0}, \pi_{2}\right)$.

\section{Analyzing a historical recovery path}

According to the bioeconomic model of the Nephrops fishery, the economic status of vessels was critical in 1994. Based on the analysis above, we can compute various possible recovery trajectories from this initial state, and compare them to the estimated historical path, used as a benchmark. In particular, we can calculate the recovery path associated with the minimum time of crisis with no constraint on transition profit (as in [29]), as well as paths where such a constraint is imposed. We compute paths where minimum transition profit are 50, 80, 90 and 100 thousand Euro respectively. The paths followed by the fishery under each of these assumptions are represented in Fig. 2 for a) fleet size and b) fishing effort, and in Fig. 3 for a) profit and b) stock biomass. 
Fleet size

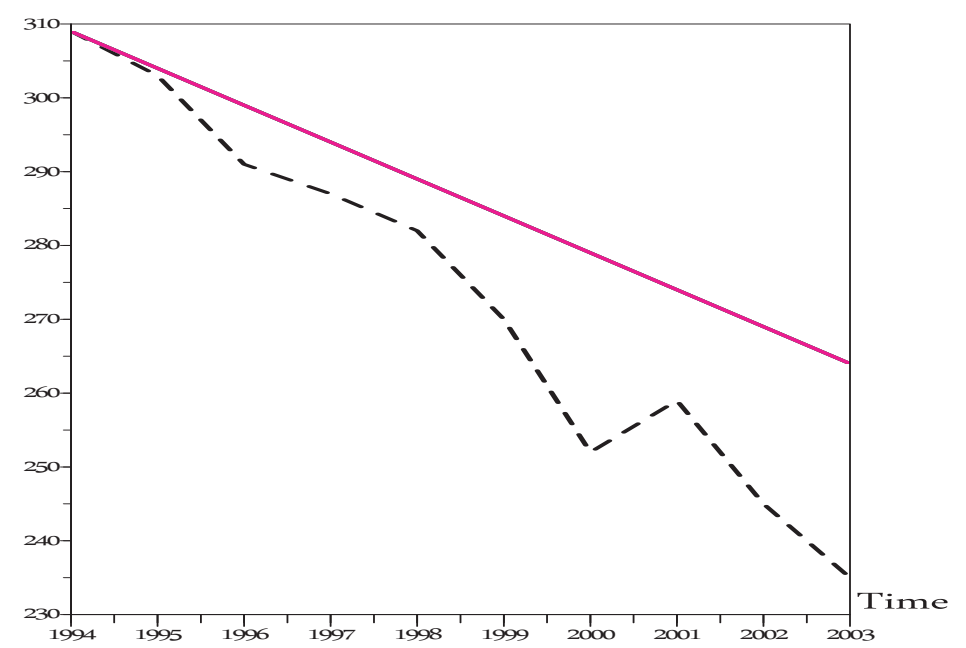

- - - Historical fleet size

Minimal time of crisis fleet size

(a) Fleet size $K_{t}$

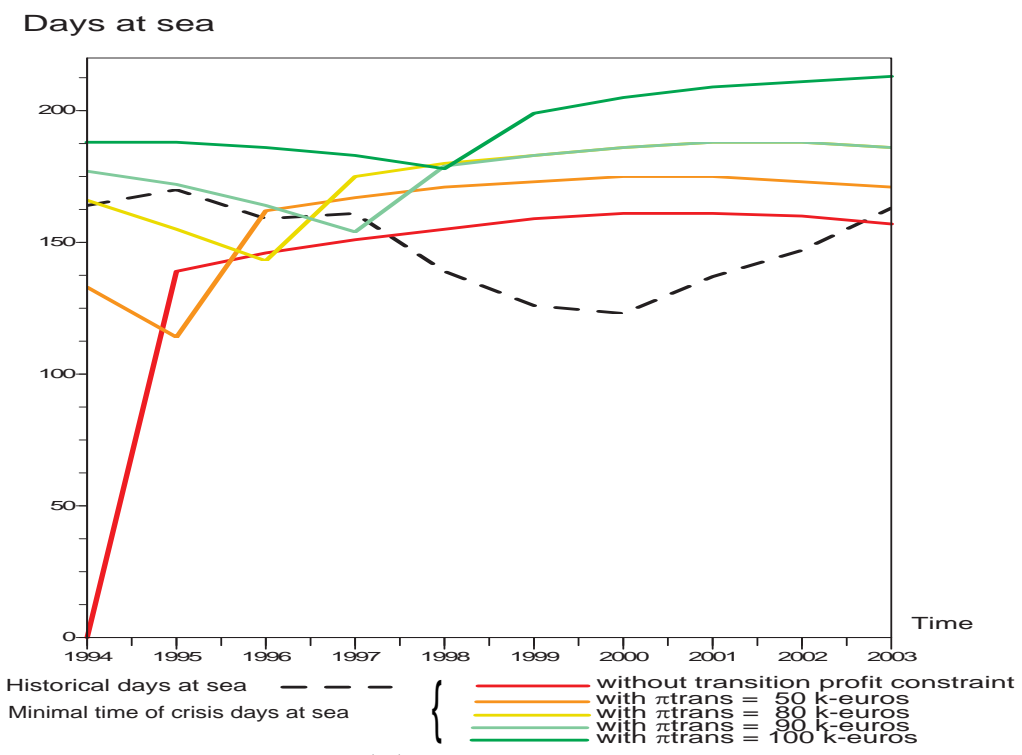

(b) Days at sea $e_{t}$

Fig. 2. Comparison of alternative recovery trajectories with different transition profit constraints 
Profit (thousands euros)

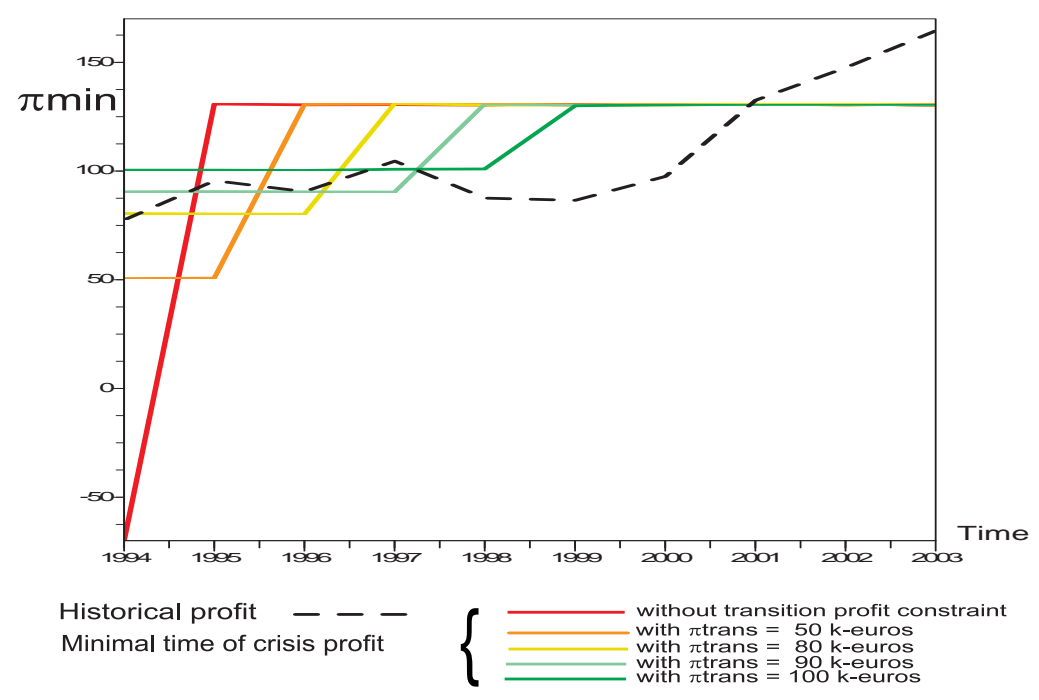

(a) Profit $\pi_{t}$

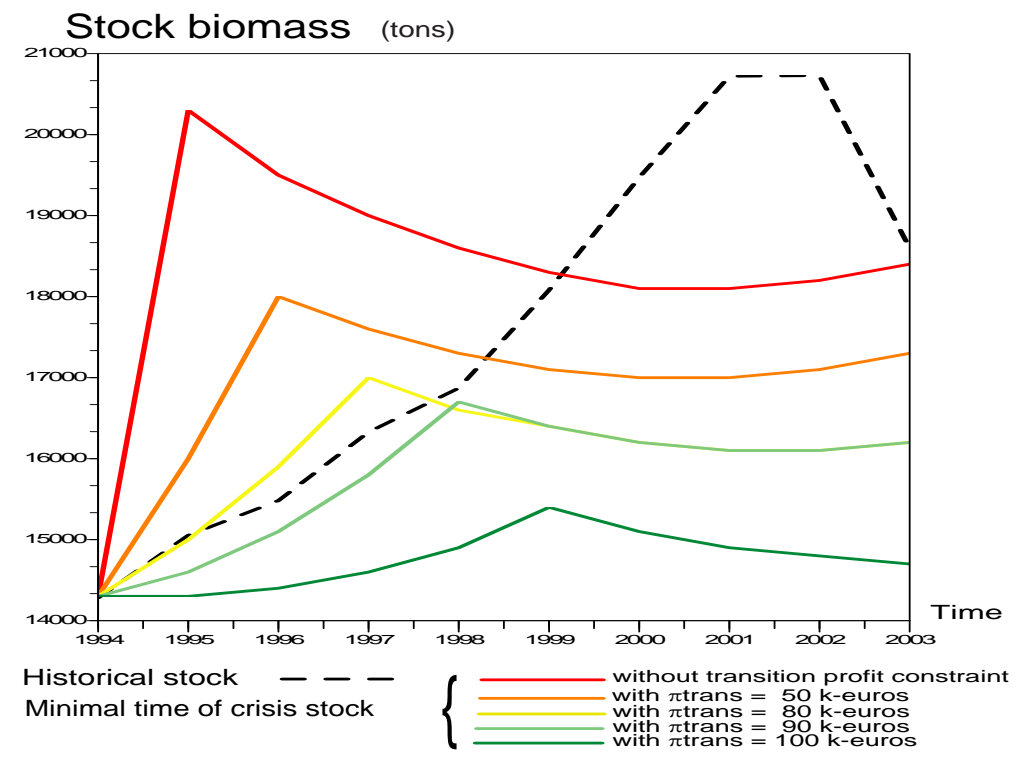

(b) Resource stock biomass $B_{t}$

Fig. 3. Comparison of alternative recovery trajectories with different transition profit constraints 
Recovering viable levels of profit with a minimum time of crisis involves reductions in fleet size (Fig. 2 panel a). The required fleet adjustment is the same whatever the constraint on transition profit. A social planner aiming to minimize the time of crisis will reduce the fleet size as much as is admissible. ${ }^{8}$ Recovery also requires a reduction of the fishing effort of vessels in the fishery (Fig. 2 panel b). This effort reduction makes stock recovery possible. However, the fishing effort reduction is constrained by the acceptability constraint $\pi_{t} \geq \pi_{\text {trans }}$. The higher this constraint, the lower the reductions of fishing effort, and the longer the time to reach the viable profit $\pi_{\min }$ (see profit pattern in Fig. 3 panel a). Moreover, the higher the transition profit, the lower the level of stock recovery (Fig. 3 panel b).

All the trajectories defined by the minimum time of crisis approach have a similar pattern, comprising two phases. In the first phase, as fishing effort decreases, the stock increases, and profit is stationary at the transition level. In the second phase, as the fishing effort increases, the stock decreases, and profit is stationary at the viability level. The switch between these phases corresponds to the end of the crisis, i.e., the moment at which the viability kernel is reached. This is the end of the recovery program, and from that moment, the fishery is said to be managed in a sustainable way.

Overall, the historical trajectory is characterized by the same temporal pattern. From 1994 to 2000, fishing effort decreased, the resource stock increased, and profit was quite stable, ranging between 80 and 100 thousand Euro. From 2001, the fishing effort increased, the resource stock decreased, and profit increased above the viability constraints. The estimated historical path of the fishery in fact appears to be comparable to the simulated recovery program minimizing the time of crisis with a transition profit of 90 thousand Euro, during the first stage of the recovery path (from 1994 to 1997). However, from 1998, the simulated program becomes viable, as it satisfies the viable profit constraint of 130 thousand Euro, whereas the estimated historical trajectory is characterized by lower level of effort per vessel, favoring stock recovery but resulting in non-viable profit levels for several extra years. The estimated historical trajectory is thus associated with a longer recovery phase. This results in higher profits per vessel at the end of the period, but with a much longer crisis period, when compared to the simulated recovery program.

Computing the recovery paths from the 1994 state for different values of the

\footnotetext{
8 Note that, in our simulations, the level $\xi_{\min }$ of the constraint on fleet size adjustment is stricter than the actual fleet size reduction observed in the fishery. Hence, our minimum time of crisis recovery scenarios are less drastic in terms of fleet reduction than what the historical trajectory. The observed cut in vessel numbers was made possible by the implementation of a public vessel buyback scheme during the period considered.
} 
transition profit constraint, we identify the minimum time of crisis associated with each of the alternative recovery programs. This allows us to quantify the trade-off between the level of guaranteed profit during the transition phase and the time needed to recover a sustainable fishery.

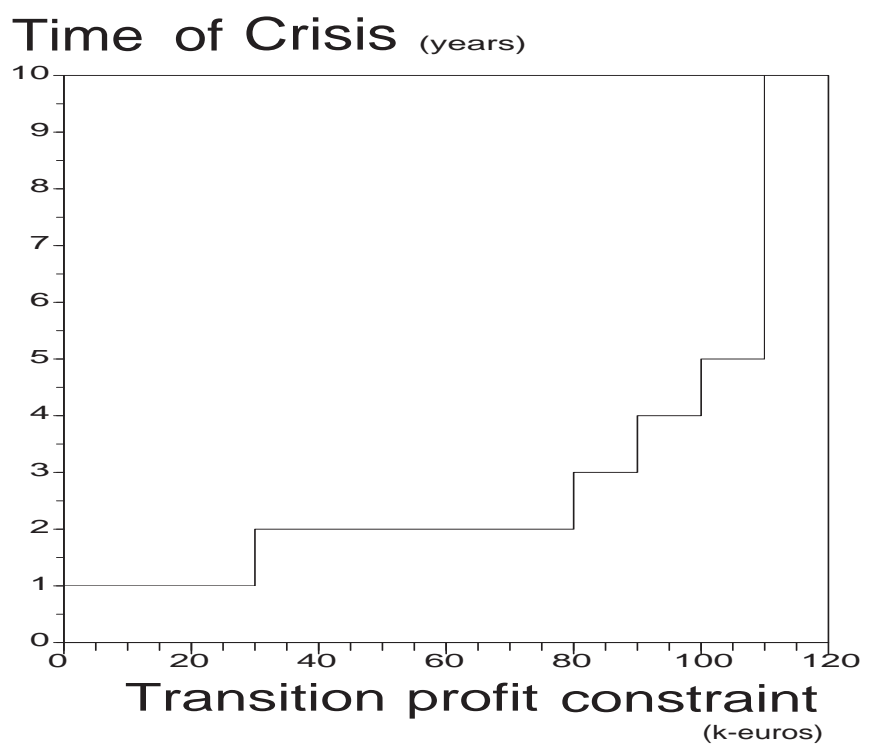

Fig. 4. Minimum time of crisis, from the 1994 state, for various transition profit constraints

Fig. 4 represents this trade-off. The greater the guaranteed profit during transition, the longer the transition. There is even a maximal transition profit constraint beyond which no recovery path for the fishery exists (infinite time of crisis). In the present analysis, this maximal transition profit is estimated to lie between 100 and 110 thousand Euro. Fig. 4 illustrates the trade-off between hare and tortoise strategies. The Hare would select a quick recovery strategy requiring a lot of sacrifices, which may not be accepted, and thus postponed. The Tortoise would select a low recovery speed, which would be well accepted and applied, but with the risk of never reaching the recovery goal in a finite time. Both of these extreme strategies may fail. Therefore, attempting to establish successful fishery restoration strategies will probably require intermediate strategies allowing compromise between speed of recovery, conflicts and the acceptability of recovery programs Where possible, the use of assessment tools, such as the one proposed here, to formalize the trade-offs involved in selecting recovery programs, may assist policy makers in identifying such compromises. 


\section{Discussion and conclusions}

It has become commonplace to describe the management of fisheries, as well as of other natural resources exploitation, as collective choice problems involving multiple objectives. In particular, a growing number of empirical studies stress the key role of political processes in explaining the observed policy choices and their consequences, and in discussing possible management scenarios. Hence, such considerations should be included in the formal description of resource system dynamics and their control. Viability analysis makes it possible to develop such an analytical approach, i.e., to consider different objectives while taking into account the inertia of dynamic bioeconomic systems.

In this paper, we propose a theoretical approach to study sustainability of bioeconomic systems in a multicriteria framework, and to define recovery paths from crisis situations. This approach is based on viability theory and its extension to the concept of minimum time of crisis. We applied this general framework to examine the viability of a fishery with respect to economic, social and biological constraints. The main constraint we considered is a minimum profit per vessel that must be guaranteed for each time period. We used the viability approach to determine the set of bioeconomic states that make it possible to satisfy the constraints dynamically. This set is called the viability kernel of the problem. Any trajectory leaving this set will violate the constraints in finite time, whatever decisions apply. The system is then facing a crisis situation. We studied transition phases from crisis situations to viable exploitation configurations. These transitions phases can be characterized by their duration and cost. This cost is defined as the difference between the minimum profit ensuring economic viability and some lower guaranteed profit during the transition phase. We showed that, if a constraint is imposed on the acceptable transition costs, this will entail longer transition phases. The reciprocal interpretation of this result is that short transition phases may not be feasible, due to the fact that they entail unacceptable short-term costs to individual operators in the fishery. Therefore, the selection of recovery paths is cast in terms of choosing between two extreme strategies. Being a Hare would imply planning for the quickest possible recovery, which requires high efforts. But as in the fable, it may be preferable to postpone efforts (fishermen may not accept the program), or to stop the recovery program before reaching the goal. Being a Tortoise would consist in limiting the transition cost and reducing recovery speed. This strategy would satisfy short term requirements of fishermen, with the risk that recovery may not be possible in finite time. The approach and methodology presented here offers a quantitative description of this trade-off between speed and acceptability.

To illustrate these theoretical results, we computed recovery paths from a historical crisis based on a simple bioeconomic model of the Nephrops fishery in 
the Bay of Biscay. We compared simulated recovery paths with the estimated historical path, and showed that the observed transition actually involved more severe adjustments than those which could have been adopted, which is probably due to the fact that public financial aid was devoted to support the adjustment of fleet capacity in France during the period studied, thus reducing transition costs.

The approach developed in this paper makes it possible to formally identify a set of sustainable states for a fishery in a multi-objective perspective, and to examine possible transition phases from unsustainable to sustainable states. The formal identification of the degree of compatibility between different constraints imposed on the management of a fishery and the dynamics of the bioeconomic system is a key result of the analysis. Based on this framework, additional research could be carried out regarding the preferred options, and trade-offs between the different objectives pursued by managers. In particular, the inclusion of costs related to the implementation of plans designed to alleviate the transition difficulties of individual operators should allow a cost-benefit analysis of management strategies. In addition, the inclusion of uncertainty in the dynamics of bioeconomic systems may lead to modified conclusions with regards to the trade-offs between speed and cost of recovery.

\section{A Mathematical details and computational methods}

Consider the criterion

$$
\mathcal{T}(X(.), u(.))=\lim _{T \rightarrow+\infty} \sum_{t=0}^{T} \mathbf{1}_{\mathcal{S}}\left(X_{t}, u_{t}\right)
$$

along trajectories $(X(),. u()$.$) such that X_{t+1}=F\left(X_{t}, u_{t}\right), u_{t} \in \mathcal{U}_{\text {trans }}\left(X_{t}\right), X_{t=0}=$ $X_{0}$.

Given the set $\mathcal{U}_{\text {trans }}(X)$ of admissible decisions from any state, we define the value function

$\mathcal{C}_{\mathcal{U}_{\text {trans }}}\left(X_{0}\right)=\inf _{u(\cdot)}\left\{\mathcal{T}(X(),. u()) \mid. X_{t+1}=F\left(X_{t}, u_{t}\right), u_{t} \in \mathcal{U}_{\text {trans }}\left(X_{t}\right), X_{t=0}=X_{0}\right\}$

The purpose of the analysis is to compute this function for all initial states (i.e. within $\mathbb{R}^{n}$ ).

We use the Dynamic Programming Principle to write the following property fulfilled by the value function $\mathcal{C}_{\mathcal{U}_{\text {trans }}}$

$$
\mathcal{C}_{\mathcal{U}_{\text {trans }}}\left(X_{0}\right)=\inf _{u_{0} \in \mathcal{U}_{\text {trans }}\left(X_{0}\right)}\left(\mathbf{1}_{\mathcal{S}}\left(X_{0}, u_{0}\right)+\mathcal{C}_{\mathcal{U}_{\text {trans }}}\left(F\left(X_{0}, u_{0}\right)\right)\right)
$$


Then, from initial condition $X_{0}$ such that $\mathcal{C}_{\mathcal{U}_{\text {trans }}}\left(X_{0}\right)<+\infty$, the optimal decisions can be easily determined, in terms of feedbacks along an optimal trajectory $\left(X^{\star}, u^{\star}\right)$

$$
u_{t}^{\star}\left(X_{t}^{\star}\right) \in \arg \min _{u_{t} \in \mathcal{U}_{\text {trans }}\left(X_{t}^{\star}\right)}\left(\mathbf{1}_{\mathcal{S}}\left(X_{t}^{\star}, u_{t}\right)+\mathcal{C}_{\mathcal{U}_{\text {trans }}}\left(F\left(X_{t}^{\star}, u_{t}\right)\right)\right), \quad t=0,1, \cdots
$$

The value function $\mathcal{C}_{\mathcal{U}_{\text {trans }}}$ can be also characterized as the fixed point of an operator.

Denote by $\mathcal{B}$ the set of bounded functions $\varphi: \mathbb{R}^{n} \mapsto \mathbb{R}$, and define the operator $\Theta: \mathcal{B} \mapsto \mathcal{B}$ such that

$$
\Theta[\varphi](X):=\min _{u \in \mathcal{U}_{\text {trans }}(X)}\left[\mathbf{1}_{\mathcal{S}}(X, u)+\varphi(F(X, u))\right]
$$

When $\mathcal{C}$ is bounded, it fulfills $\Theta[\mathcal{C}]=\mathcal{C}$. In addition, if the operator $\Theta$ is a contraction mapping, the fixed point can be determined by the contraction mapping theorem (see [3]). Unfortunately, this is not the case for the operator defined in (A.5) and one cannot guarantee that $\mathcal{C}$ is bounded.

Instead, one can consider the discounted operator

$$
\Theta_{\theta}[\varphi](X):=\min _{u \in \mathcal{U}_{\text {trans }}}\left[\mathbf{1}_{\mathcal{S}}(X, u)+\theta \varphi(F(X, u))\right]
$$

where $0<\theta<1$ is the contraction parameter that guarantees the convergence of the Picard algorithm

$$
\mathcal{C}_{\theta}=\lim _{n \rightarrow+\infty} \Theta_{\theta}^{n}\left[\varphi_{0}\right]
$$

from any initial function $\varphi_{0} \in \mathcal{B}$. Note that the fixed point $\mathcal{C}_{\theta}$ of the operator $\Theta_{\theta}$ is the value function

$$
\mathcal{C}_{\theta}\left(X_{0}\right)=\inf _{u(\cdot)}\left\{\mathcal{T}_{\theta}(X(.), u(.)) \mid X_{t+1}=F\left(X_{t}, u_{t}\right), u_{t} \in \mathcal{U}_{\text {trans }}\left(X_{t}\right), X_{t=0}=X_{0}\right\}
$$

for the discounted criterion

$$
\mathcal{T}_{\theta}(X(.), u(.))=\lim _{T \rightarrow+\infty} \sum_{t=0}^{T} \theta^{t} \mathbf{1}_{\mathcal{S}}\left(X_{t}, u_{t}\right)
$$

Note also that the convexity of the functions $f(\theta)=\theta^{n}$ for $\theta>0$ implies the property $\theta^{n}=f(\theta) \geq f(1)+f^{\prime}(1)(\theta-1)=1-n(1-\theta)$.

Consider a trajectory $(X(),. u()$.$) such that \mathcal{T}(X(),. u())<.+\infty$. Then, there exists $T \geq 0$ such that $\theta^{t} \mathbf{1}_{\mathcal{S}}(X, u)=0$ for any $t>T$, and one has the following inequalities:

$$
\mathcal{T}(X(.), u(.))-(1-\theta) \frac{T(T-1)}{2} \leq \mathcal{T}_{\theta}(X(.), u(.)) \leq \mathcal{T}(X(.), u(.))
$$


from which we deduce

$$
\lim _{\theta \rightarrow 1} \mathcal{T}_{\theta}(X, u)=\mathcal{T}(X, u),
$$

and the approximation of the value function $\mathcal{C}$ by $\mathcal{C}_{\theta}$ with $\theta$ close to 1

$$
\mathcal{C}_{\mathcal{U}_{\text {trans }}}\left(X_{0}\right)<+\infty \Rightarrow \lim _{\theta \rightarrow 1} \mathcal{C}_{\theta}\left(X_{0}\right)=\mathcal{C}_{\mathcal{U}_{\text {trans }}}\left(X_{0}\right)
$$

Note also that the function $\mathcal{C}_{\theta}$ is bounded

$$
\left\|\mathcal{C}_{\theta}\right\|_{\infty}=\max _{X_{0}} \mathcal{C}_{\theta}\left(X_{0}\right) \leq \frac{1}{1-\theta} .
$$

Denote by $V_{\theta}^{n}$ the n-th iterations of the Picard algorithm

$$
V_{\theta}^{n}=\Theta_{\theta}^{n}\left[\varphi_{0}\right] .
$$

From the contraction property of the operator $\Theta_{\theta}$, one has

$$
\left\|V_{\theta}^{n}-\mathcal{C}_{\theta}\right\|_{\infty} \leq \theta^{n}\left\|\varphi-\mathcal{C}_{\theta}\right\|_{\infty}^{n}
$$

Choosing $\varphi_{0} \equiv 0$, one obtains the error estimation of the n-th iteration

$$
\left\|V_{\theta}^{n}-\mathcal{C}_{\theta}\right\|_{\infty} \leq \frac{\theta^{n}}{1-\theta} .
$$

Let us assume, from the computational point of view, that we are interested in approximations of the optimal trajectories over $T$ first time steps only. This means that the computation accuracy of the criterion $\mathcal{T}_{\theta}$ or the value function $\mathcal{C}_{\theta}$ should be less than $\theta^{T+1}$. If $a$ is the machine accuracy, $\theta$ should be chosen such that $\theta^{T+1}>a$, and the approximation $V_{\theta}^{n}$ of the value function $\mathcal{C}_{\theta}$ should be realized with an error that fulfills the inequality

$$
\left\|V_{\theta}^{n}-\mathcal{C}_{\theta}\right\|_{\infty}<\theta^{T+1}
$$

This condition is guaranteed for a number of iterations

$$
n>T+1+\frac{\log (1-\theta)}{\log \theta}
$$

For an accuracy $a=10^{-6}$, the following values are admissible.

\begin{tabular}{|c|ccccc|}
\hline $\mathrm{T}$ & 5 & 10 & 20 & 100 & 1000 \\
\hline$\theta$ & 0.1 & 0.3 & 0.6 & 0.9 & 0.99 \\
\hline$n$ & 6 & 11 & 22 & 123 & 1460 \\
\hline
\end{tabular}




\section{B Data and parameters}

The analysis is applied to the Bay of Biscay Nephrops fishery (ICES area VIII), which is fished by French trawlers based in ports of the Atlantic coast of France. We used model calibration results of Martinet et al. [29], the methodology of which is briefly summarized below.

Stock growth model: Available data on landings, technical characteristics and fishing effort of the trawler fleet, as well as estimates of stock abundance and total catch of this fleet provided by the ICES working group on Nephrops for the 1987-2003 period was used to calibrate the biological parameters of the logistic growth function (eq. 8). Landings Per Unit of Effort (LPUE) were used as an index of Nephrops stock abundance, and calibration of the parameters was carried out using estimation methods proposed by [21], with observed LPUE of the total fleet over the study period as a reference. Nonlinear parameter estimation was performed to find the best fit of the model, with the minimization of the squared deviation between the logged values of observed and predicted LPUE as the fitting criterion ([21]). Parameter values corresponding to the best fit are presented in the table at the end of this appendix. (See [29] for the comparison of observed versus predicted LPUE for the adjusted fleet, using the parameters derived from this calibration procedure.)

The discard rate used in the analysis was the average estimated discard rate over the study period as described in reports of the ICES working group on the Bay of Biscay Nephrops stock. ${ }^{9}$

Economic parameters were estimated using costs and earnings data collected by the Fisheries Information System of Ifremer via surveys of individual vessel owners (https://www.ifremer.fr/isih). We used estimates of average annual gross returns and costs produced by the information system, for the different categories of trawlers considered in the analysis, for years 2001-2003. Estimation of the average annual fixed costs used in the analysis includes the costs of maintenance and repair, social contributions by vessel owners, insurance costs, tax paid to professional organizations, and license costs. Estimation of average annual variable costs includes landing charges, oil and fuel costs, and food and ice costs. The price used in the analysis was the average ex-vessel price per ton of landings at the Nephrops fishery as observed over the study

$\overline{9}$ Technical reports are :

- ICES (2003a) Report of the Working Group on the assessment of southern shelf stocks of Hake, Monk and Megrim. ICES CM 2004/ACF:02.

- ICES (2003b) Report of the working group on Nephrops Stocks, ICES CM 2003/ACFM:18. 
period.

The minimum profit constraint was defined as the minimum level of profit ensuring remuneration of the productive factors at their opportunity cost. The opportunity cost of capital was defined as the observed average interest rate for capital remuneration in recent years in the French context; a value of .04 was used in the analysis. With an average capital value of vessels estimated at 348,000 Euro, the opportunity cost of capital was estimated at approximately 14,000 Euro. The opportunity cost of labor was fixed at the average level of remuneration of labor in the fishery in years 2001-2003. It has been stressed that this was barely sufficient to attract fishermen to the fishery. Based on the available data, the opportunity cost of labor was fixed at 35,000 Euro per fisher per year; with an average crew size of 3.3 men, this amounted to a total opportunity cost of labor of approximately 116,000 Euro per year per vessel.

The share of revenue related to Nephrops fishery catches was fixed at the average share ob- served over the study period, and was assumed constant; hence we assume that revenue derived from other species will vary in direct proportion with the revenue derived from Nephrops landings.

Finally, the speed at which the fleet was allowed to adjust (+/- 5 vessels per year maximum) was fixed in order to reflect the variations in fleet size under normal conditions of exploitation, based on the available data ([38]).

The table below summarizes the parameter values used in the analysis.

\section{Parameters of the case study: the Bay of Biscay Nephrops fishery (ICES area VIII)}

Parameters values are as follows.

$$
\begin{aligned}
\text { Parameter } & \text { value } \\
r= & 0.78 \\
B_{\text {sup }} & =30,800 \text { tons } \\
q & =72.10^{-7} \mathrm{j}^{-1} \\
p & =8,500 \text { Euro per tons } \\
\omega_{f} & =70,000 \text { Euro per year } \\
\omega_{v} & =377 \text { Euro per day at sea } \\
e_{\text {sup }} & =220 \text { days } \\
\tau_{d}= & 33 \% \\
\lambda= & 43 \%
\end{aligned}
$$


Acknowledgments This research work was carried out as part of the CHALOUPE research project (www.projet-chaloupe.fr), funded by the French National Research Agency as part of its "Biodiversity" program. We are most grateful to Luc Doyen, the editor and two referees for helpful comments. All errors are our own.

\section{References}

[1] Aubin, J.-P. (1991). Viability theory. Birkhauser, Springer Verlag.

[2] Béné, C., Doyen, L., Gabay, D. (2001). A viability analysis for a bio-economic model. Ecological Economics, 36, 385-396.

[3] Bertsekas, D. (1987). Dynamic Programming: Deterministic and Stochastic Models, Prentice-Hall.

[4] Charles, A. (1994). Towards sustainability: the fishery experience. Ecological Economics, 11, 201-211.

[5] Cheung, W.W.L., Sumaila, U.R. (2007). Trade-offs between conservation and socio-economic objectives in managing a tropical marine ecosystem. Ecological Economics, 66, 193-210.

[6] Chichilnisky, G. (1977). Economic development and efficiency criteria in the satisfaction of basic needs. Applied Mathematical Modelling, 1, 290-298.

[7] Clark, C.W. (1985). Bioeconomic Modelling and Fisheries Management. John Wiley and Sons: New York, 1985.

[8] Clark, C.W. (1990). Mathematical Bio-economics: the optimal management of renewable resources. Second edition, John Wiley and Sons: New York.

[9] Clark, C.W., Munro, G.R., Sumaila, U.R. (2005). Subsidies, buybacks, and sustainable fisheries. Journal of Environmental Economics and Management, 50, $47-58$.

[10] Cochrane, K. (2000). Reconciling sustainability, economic efficiency and equity in fisheries: the one that got away? Fish and Fisheries, 1, 3-21.

[11] Cury, P., Mullon, C., Garcia, S., Shannon, L.J. (2005). Viability theory for an ecosystem approach to fisheries, ICES Journal of Marine Science, 62, 577-584.

[12] De Lara, M., Doyen, L. (2008). Sustainable Management of Natural Resources: Mathematical Models and Methods. Springer, 266p.

[13] Doyen, L., Saint-Pierre, P. (1997). Scale of viability and minimum time of crisis. Set-valued Analysis, 5 ,227-246.

[14] Eisenack, K., Sheffran, J., Kropp, J. (2006). The Viability Analysis of Management Frameworks for fisheries. Environmental Modeling and Assessment, 11, 69-79. 
[15] Food and Agriculture Organization (2004). The state of World Fisheries and Aquaculture. FAO, Sofia.

[16] Garcia, S., Grainger, J.R. (2005). Gloom and doom? The future of marine capture fisheries. Phil. Trans. R. Soc. B., 360, 21-46.

[17] Gordon, H.S. (1954). The economic theory of a common property resource: the fishery. Journal of Political Economy, 82, 124-142.

[18] Groot, J., Rossing, W., Jellema, A., Stobbelaar, D.J., Renting, H., Van Ittersum, M. (2007). Exploring multi-scale trade-offs between nature conservation, agricultural profits and landscape quality - A methodology to support discussions on land-use perspectives. Agriculture, Ecosystems \& Environment, 120, 58-69.

[19] Hannesson, R. (2007). Growth accounting in fishery. Journal of Environmental Economics and Management, 53, 364-376.

[20] Hilborn, R. (2007). Defining success in fisheries and conflicts in objectives. Marine Policy, 31, 153-158.

[21] Hilborn, R., Walters, C. (1992). Quantitative Fisheries Stock Assessment: Choice, Dynamics and Uncertainty. International Thomson Publishing.

[22] Holland, D.S. (2000). A bioeconomic model for marine sanctuaries on Georges Bank. Journal of Fisheries and Aquatic Sciences, 57, 1307-1319.

[23] Hutton, T., Guyader, O., Prellezo, R., Mardle, S., Thébaud, O. (2006). An analysis of changing fishing units using field surveys: case studies from the E.U., In Proceedings of the 13th biennial conference of the IIFET, Portsmouth, UK.

[24] Jorgensen, C., et al. (2007). Managing Evolving Fish Stocks. Science 318 (November 23), 1247-1248.

[25] Krawczyk, J., Kunhong, K. (2009). Satisficing solutions to a monetary policy problem. Macroeconomic Dynamics, 13, 46-80.

[26] Mardle, S., Pascoe, S. (1999). A Review of Applications of Multiple-Criteria Decision-Making Techniques to Fisheries. Marine Resource Economics, 14, 41-63.

[27] Mardle, S., Pascoe, S. (2002). Modeling the effects of trade-offs between long run and short run objectives in the North Sea. Journal of Environmental Management, $65,49-62$.

[28] Martinet, V., Doyen, L. (2007). Sustainability of an economy with an exhaustible resource: a viable control approach. Resource and Energy Economics, 29, 17-39.

[29] Martinet, V., Thébaud, O., Doyen, L. (2007). Defining viable recovery paths toward sustainable fisheries. Ecological Economics, 64, 411-422.

[30] Millennium Ecosystem Assessment (2005). Ecosystems and Human well-being: general synthesis. Washington D.C., Island Press, pp.160. 
[31] Morris, W.F., Doak, D.F. (2003). Quantitative Conservation Biology: Theory and Practice of Population Viability Analysis. Sinauer Associates.

[32] Polasky, S., Nelson, E., Lonsdorft, E., Fackler, P., Starfield, A. (2005). Conserving species in a working landscape: land use with biological and economic objectives. Ecological Applications, 15, 1387-1401.

[33] Schaefer, M.B. (1954). Some aspects of the dynamics of populations. Bull. Int. Am. Trop. Tuna Comm., 1, 126-156.

[34] Sethi, G., Costello, C., Fisher, A., Hanemann, M., Karp, L. (2005). Fishery management under multiple uncertainty. Journal of Environmental Economics and Management, 50, 300-318.

[35] Simon, H. (1957). A Behavioral Model of Rational Choice, in Models of Man, Social and Rational: Mathematical Essays on Rational Human Behavior in a Social Setting. New York: Wiley.

[36] Singh, R., Weninger, Q., Doyle, M. (2006). Fisheries management with stock growth uncertainty and costly capital adjustment. Journal of Environmental Economics and Management, 52, 582-599.

[37] Smith, V. (1969). On Models of Commercial Fishing. Journal of Political Economy, 77, 181-196.

[38] Thébaud, O., Daurès, F., Guyader, O., Travers, M., Van Iseghem, S. (2006). Modeling the adjustment of fishing fleets to regulatory controls: the case of SouthBrittany trawlers (France), 1990-2003. AMURE Working paper series D-13-2006.

[39] United Nations (2002). Report of the World Summit on Sustainable Development. Johannesburg. 